\title{
QoS Oriented Cross-synch Routing Protocol for Event Driven, Mission-critical Communication Over MANET: Q-CSRPM
}

\author{
Shashi Raj K \\ Department of ECE, DSCE, Bangalore \\ E-mail: shashiraj18@gmail.com \\ Siddesh G K \\ Department of ECE, JSSATE, Bangalore \\ E-mail: siddeshgundagatti@gmail.com
}

Received: 25 July 2018; Accepted: 05 September 2018; Published: 08 November 2018

\begin{abstract}
The decentralized and infrastructure less feature of Mobile Ad-hoc network (MANET) has made it a potential networking solution to be used in major applications ranging natural disaster management, vehicular communication, industrial communication etc. Though, being a dominating mobile communication system, exceedingly high network topology and mobility pattern in MANETs make it trivial to achieve Quality of Service (QoS) delivery, particularly for event-driven (mission-critical) communication. With this motivation, in this research paper a robust QoS Oriented Cross-Synch Routing Protocol for Event Driven, Mission-Critical Communication named Q-CSRPM has been developed for MANET. The proposed Q-CSRPM routing protocol exploits cross-layer routing architecture by applying network layer, MAC layer and physical layer information of IEEE 802.11 standard to perform optimal best forwarding node selection and reliable path formation. QCSRPM protocol performed proactive node management, service differentiation based data prioritization and resource scheduling, and dynamic buffer assessment based congestion detection at the network layer, dynamic link quality estimation and packet velocity estimation at the MAC layer, and PHY switching control at the physical layer of the protocol stack. Q-CSRPM applies dynamic link quality, congestion probability and packet velocity of a node for best forwarding node selection to form forwarding path. The node information sharing across the layers of protocol stack enables optimal BFN selection and routing control. It strengthened Q-CSRPM to exhibit $98.2 \%$ and $93 \%$ of packet delivery ratio for real time data and non-real time data respectively. A minimum of $2 \%$ deadline miss ratio was observed.
\end{abstract}

Index Terms - MANET, Quality of Service, Cross-Layer Design, Mission critical communication.

\section{INTRODUCTION}

In last few years, global human presence has witnessed exponentially rising development in communication systems, particularly wireless networks to meet major communication-demands. This high pace increase in communication technologies and associated applications have motivated academia-industries to develop certain more efficient routing protocols to meet emerging Quality of Service (QoS) demands under varied network conditions. Among major wireless communication systems, MANET [1] has emerged as one of the best suitable infrastructure-less and decentralized networks having immense potential to serve communication demands by incorporating sensor or node mobility in adhoc manner. In practice, MANETs are constituted dynamically with the collection of randomly placed wireless mobile nodes without incorporating any complicated infrastructure that eventually reduces set-up time significantly. In typical communication environment, some or even all nodes of MANET can function as routers to perform communication between two hosts by incorporating multi-hop routing. In general, to constitute MANET various devices like mobile phones, laptops, pocket-computers with wireless connectivity are applied. Being a decentralized and infrastructure less network MANET undergoes significantly high topological changes that as a result make QoS efficient, reliable transmission difficult. In practice, the changing network topology influences key network parameters such as link quality, link outage probability, (per node) resource availability and packet velocity etc. These factors often affect reliability of the data transmission and QoS assurance in MANET. Though, all the comprising nodes in MANETs maintain network connectivity in a cooperative manner without applying any fixed infrastructure such as additional access points or base stations, mobility affects node characteristics and overall transmission efficacy. The individual node in MANETs possesses a defined routing protocol to perform data communication or forwarding to the next hop (intermediate) nodes. Under mobile topology if two nodes are in communication range they can directly 
communicate to each other, otherwise nodes forward their data to the next hop node to ensure timely and reliable data transmission. Though, a few efforts have been made on exploiting dynamic network parameters such as residual energy, signal to noise ratio, inter-node distance, link quality etc as individual parameter to perform routing decision; however applying single network parameter even under dynamic topology can't assure reliable communication. For instance, applying merely inter-node distance as routing decision parameter may face a problem when even though a node is within radio-range it might be scarce of buffer space to accommodate forwarding data and hence can't be suitable to forward data towards destination. Similarly, even thou a node can have enough buffer space but very less transmission rate (say, packet injection rate) might take more time to transmit the data, which could violate deadline sensitive transmission needs. These facts signifies that to make a suitable and optimal routing decision knowing multiple node/network parameters can be vital that consequently could help in exploiting those network parameters from the different layers of the protocol stack to make suitable routing decision. This approach of applying multiple (dynamic) network parameters for routing decision is called Cross-Layer Architecture (CLA) [2,3]. Undeniably, a number of efforts have been made on improving MANET routing protocol [4-8], however as per our current knowledge no significant effort is made on exploiting dynamic network states parameters obtained from the different layers of the protocol to perform route decision. In majority of works, either single parameter such as residual energy, internode distance etc are applied to perform routing. Exploiting other parameters such as adaptive inter-node link quality, packet velocity, (per node) resource availability etc can ensure a potential routing protocol for MANETs. To achieve QoS centric and reliable communication, ensuring network topology awareness based Best Forwarding Node (BFN) selection can be of paramount significance. This research mainly focuses on developing a novel cross layer architecture (CLA) based routing protocol for QoS-centric communication over MANET. In this research a novel CLA based QoS centric routing protocol is developed by exploiting dynamic parameters from the different layers of the IEEE 802.11a protocol stack, where key node parameters such as packet velocity, dynamic link quality, cumulative congestion degree etc have been applied to decide a node to become $\mathrm{BFN}$ for reliable and deadline sensitive transmission. The overall proposed system is developed using MATLAB $2012 \mathrm{~b}$ simulation platform and performance is measured in terms of throughput, packet loss and deadline miss ratio which are vital to assure QoS transmission. The list of key variables used in this manuscript and their definitions is given in Table 1 .

Table 1. List of Abbreviations

$\begin{array}{ll}\text { Abbreviation } & \text { Definition } \\ \text { MANET } & \text { Mobile Ad-hoc network } \\ \text { QoS } & \text { Quality of Service } \\ \text { NRT } & \text { Non-Real-Time }\end{array}$

\begin{tabular}{|c|c|}
\hline RTD & Real Time Data \\
\hline $\mathrm{BFN}$ & Best Forwarding Node \\
\hline CLA & Cross-Layer Architecture \\
\hline Q-CSRPM & $\begin{array}{l}\text { QoS oriented Cross-Synch Routing Protocol } \\
\text { to be used for event driven, mission-critical } \\
\text { communication over MANETs }\end{array}$ \\
\hline SD & Service Differentiation \\
\hline DCD & Dynamic Congestion Detection \\
\hline LQE & Link Quality Estimation \\
\hline FA & Filling Area \\
\hline AOMDV & $\begin{array}{l}\text { Ad Hoc On Demand Multipath Distance } \\
\text { Vector }\end{array}$ \\
\hline GA & Genetic Algorithm \\
\hline $\mathrm{CH}$ & Cluster Head \\
\hline RMA & Rate Monotonic Algorithm \\
\hline EDF & Earliest Deadline First \\
\hline HCLP & Hierarchical Cross Layer Optimization \\
\hline XLO & XL Optimizer \\
\hline SDRA & $\begin{array}{l}\text { Service Differentiation and Resource } \\
\text { Allocation }\end{array}$ \\
\hline PDR & Packet Delivery Ratio \\
\hline CNRM & Cumulative Node Rank Matrix \\
\hline FIFO & First in First out \\
\hline $\mathrm{CI}$ & Congestion Index \\
\hline MRTT & Mean Round Trip Time \\
\hline DPM & Dynamic Power Control \\
\hline CLAODV & Cross layer based AODV \\
\hline $\mathrm{AMC}$ & Adaptive Modulation and Coding \\
\hline DMR & Deadline Miss Ratio \\
\hline CNRM & Cumulative Node Rank Matrix \\
\hline Node $_{\text {Table }}$ & Node table \\
\hline $\mathrm{BFN}_{\mathrm{i}}$ & Feasible BFN of the ith node \\
\hline $\mathrm{Neigh}_{\mathrm{j}}$ & One-hop distant neighbour of the ith node \\
\hline $\mathrm{Eucl}_{\mathrm{d}}$ & $\begin{array}{l}\text { Euclidean distance between the BFN and the } \\
\text { source node }\end{array}$ \\
\hline Eucl $_{\mathrm{F}}$ & $\begin{array}{l}\text { Euclidean distance between the BFN and the } \\
\text { nearest destination node }\end{array}$ \\
\hline $\mathrm{T}_{\mathrm{d}_{-} \mathrm{i}}$ & Remaining deadline time of the packet \\
\hline $\mathrm{d}_{\mathrm{i}}^{\mathrm{j}}$ & $\begin{array}{l}\text { Euclidian distance between the forwarding } \\
\text { node } i \text { and the nearest sink } \mathrm{j}\end{array}$ \\
\hline $\mathrm{S}_{\mathrm{n}}$ & Node subset \\
\hline $\mathrm{CI}_{\text {NRTBuffer }}$ & $\begin{array}{l}\text { Buffer or memory available in NRT related } \\
\text { normal queue }\end{array}$ \\
\hline $\mathrm{CI}_{\text {RTDBuffer }}$ & $\begin{array}{l}\text { Buffer or memory available in RTD related } \\
\text { buffer in prioritized queue }\end{array}$ \\
\hline $\mathrm{CI}_{\text {NRTBufferMax }}$ & Maximum buffer capacity of the NRT \\
\hline $\mathrm{CI}_{\mathrm{RTDMax}}$ & Maximum buffer capacity of the RTD \\
\hline $\mathrm{CI}_{\mathrm{r}}$ & Cumulative congestion degree \\
\hline$\Psi$ & Dynamic link quality \\
\hline A & Network adaptive constant parameter \\
\hline $\mathrm{N}_{\mathrm{rx}}$ & Total number of the received packets \\
\hline $\mathrm{N}_{\mathrm{tx}}$ & Total number of the transmitted packets \\
\hline $\mathrm{S}_{\mathrm{t}}$ & Speed of radio signal \\
\hline $\mathrm{P}_{\mathrm{Vel}}$ & $\begin{array}{l}\text { Maximum rate of data transmission of a node } \\
\text { or packet velocity }\end{array}$ \\
\hline $\mathrm{Tp}$ & Transmission power \\
\hline $\mathrm{D}_{\mathrm{ESD}}^{\mathrm{i}}$ & $\begin{array}{l}\text { Euclidean distance between source node } \mathrm{i} \text { and } \\
\text { the sink node }\end{array}$ \\
\hline $\mathrm{D}_{\mathrm{ENS}}^{\mathrm{i}}$ & $\begin{array}{l}\text { Euclidean distance in between source and the } \\
\text { nearest sink }\end{array}$ \\
\hline $\mathrm{ARTT}_{\mathrm{Ti}}$ & Average round trip time \\
\hline $\mathrm{R}_{\text {MaxSpeed }}$ & Maximum speed of the radio signal in air \\
\hline $\mathrm{R}_{\text {At }}^{\mathrm{i}}$ & Time of ACK receiving \\
\hline$S_{\mathrm{pt}}^{\mathrm{i}}$ & Time of packet transmission \\
\hline $\mathrm{N}$ & Total number of packets transmitted \\
\hline $\mathrm{W}_{1}$ & Weight parameter assigned to link quality \\
\hline $\mathrm{W}_{2}$ & $\begin{array}{l}\text { Weight parameter assigned to congestion } \\
\text { degree }\end{array}$ \\
\hline $\mathrm{W}_{3}$ & Weight parameter assigned to packet velocity \\
\hline CNRM & Cumulative node-score matrix for ith node \\
\hline Current $_{\text {CRM }}$ & Current rank matrix \\
\hline
\end{tabular}

The other sections of the presented manuscript present 
the following. Section II discussed related wok, which is followed by the discussion of the proposed method in Section III. Results obtained are discussed in Section IV and the overall conclusion of the presented research work is given in Section V. References used in this paper are presented at the end of manuscript.

\section{RELATED WORK}

As stated in previous section, Ad-hoc network is a type of decentralized and infrastructure less network that exhibits communication over wireless media, primarily by means of the radio propagation in air. Since, Ad-hoc network performs communication between neighboring nodes and hence employs multihop transmission to perform data communication. Undeniably, traversal over multiple hops often influences overall QoS provision due to direct affect on end-to-end delay, latency, packet loss probability, etc. On the other hand, mobility in MANET often imposes link vulnerability or link outage probability. Under such circumstances, enabling QoS is difficult that even becomes more trivial due to dynamic topology variations and inherent network information. Considering significance of MANET and associated limitations, a number of efforts have been done to achieve certain more efficient and QoS centric routing protocol. Some of the key potential efforts made are routing optimization at the different layers of the protocol stack [9-11]. Authors [9-11] emphasized on achieving QoS provision by enabling timely and reliable data transmission. However, to achieve reliable data transmission two dominant approaches, retransmission and replication have been applied [12]. To achieve higher reliability for QoS provision authors [13] focused on multi-path transmission. Authors developed a routing protocol named Sequential Assignment Routing (SAR) [13] that forms multipath in between source to the destination by forming multiple trees in which the root of the individual tree is a one-hop neighbor. To perform QoS centric routing authors considered energy of each path and data priority. The route with maximum energy was selected as best forwarding node. A similar effort was made in [14] where in addition to the energy of each path authors applied end-to-end delay to optimize throughput for best effort data traffic. Authors [14] assumed that the each node possesses a data-traffic classifier that classifies data into RTD and NRT and prioritizes accordingly. In [15] a multipath routing protocol was developed. A link quality based energy efficient routing protocol for MANET was developed in [15] where a connectivity graph was formed based on minimum hop counts and maximum link quality. A cross-layer routing model was developed in [3] where once assigning application authors estimated cost of each path in between source and the sink. To meet QoS demands in MANET, authors considered node-entropy as a parameter to perform route decision. Authors applied bandwidth reservation policy and node-entropy as the decision variable to perform routing decision. In [16], authors considered MANET with definite buffer size and network lifetime to augment performance. Authors exploited the concept of Markov chain that enables feasible and input rate-dependent throughput and packet loss ratio even under dynamic transmission scenario. Authors applied M/G/1/K queuing concept, end-to-end delay to assess the impact of deadline time (packet's endto-end delay) and buffer size on network throughput, delay and packet loss. Authors in [17] developed a multicast routing protocol for MANET that forms multiple multicast trees in which the individual multicast tree can meet a predefined bandwidth demands to ensure reliable transmission. Authors applied network coding to assure resource conservation and reliable transmission.

Researchers applied kNN query processing concept in MANET to enable efficient query classification under dynamic network topology [18]. In their model Filling Area (FA) model was applied to process kNN queries that assured low overhead and minimal search space. Authors exploited node-statistics (i.e., delay and bandwidth occupancy) under mobility to perform MANET routing [19]. In [20] authors emphasized on energy conservation in MANET by applying energy consumption as objective function of Ad Hoc On Demand Multipath Distance Vector (AOMDV) routing protocol. In this model, authors considered fitness function to obtain the best path from source node to destination node for achieving minimum energy consumption in multipath routing. Considering dynamic nature of topology in MANET's authors [21] found energy replacement infeasible and hence proposed clustering based model armored with soft computing algorithms for clustering formation. Authors suggested an evolutionary computing algorithm named Genetic Algorithm (GA) for $\mathrm{CH}$ selection, where a node with maximum reliability or network rank was considered as objective function to be achieved. Clustering based secure and efficient routing protocol for MANET was developed in [22]. Unlike classical security models such as encryption or firewalls authors derived a novel approach based on node trustworthiness. To augment routing performance authors applied battery power, each node trust value and mobility as decision parameter for Cluster Head $(\mathrm{CH})$ selection. A similar effort was made in [23] where authors proposed a weight based clustering model which was developed on the basis of the cumulative weight metric obtained for different parameters such as inter-node distance, radio range of the node and residual energy. To avoid any intrusion in the network authors [23] proposed network partitioning concept. In [24] authors developed a QoS centric routing protocol using cross-layer concept armored with real time scheduling at the network layer with enhanced Rate Monotonic Algorithm (RMA) and Earliest Deadline First (EDF). In [25] authors applied cross layer architecture where they considered node density, mobility (i.e., topological variation), and MAC queue utilization so as to ensure channel state awareness for QoS centric communication. A cross layer model was developed in [26] where authors developed underlay model at the network layer of the protocol stack (IEEE 802.11). To 
minimize the issue of flooding overhead they developed a cross-layered location restricted energy efficient routing approach. This approach ensured optimal end-to-end guarantee at the transport layer. An alternative of AODV/DSR protocols was developed in [27]. A design of cross-layer framework between application and MAC and between MAC and transport layer was proposed in [28]. Authors [29] emphasized on developing a joint optimization model in between MAC layer and the application layer that enabled mapping of video frames on the basis of its priority and network traffic load. To augment QoS provision and reliable communication, authors [29] developed a multipath DSR-based routing protocol. This approach incorporated a load balancing model that reduced end-to-end delay and data drop probability and decreasing the end-to-end delay. Crosslayer architecture based MANET routing protocol, ViStA-XL was developed in [30] where author enhanced it to enable efficient video-streaming services. In their proposed model a real-time XL Optimizer (XLO) was incorporated to gather node information and network status information from the different layers of the network's protocol stack. To reduce error between received and transmitted video, XLO component performs dynamic decision for the different layers of the protocol stack. Authors [31] exploited cross-layer design concept to develop a distributed energy-adaptive location-based CMAC protocol named DEL-CMAC for MANETs. QoS centric routing model was developed in [32]. Authors [33] too developed a Hierarchical Cross Layer Optimization (HCLP) routing protocol to achieve high resource utilization, minimum delay and minimum jitter in MANET.

\section{PROBLEM FORMULATION}

To ensure QoS provision and reliable data communication over MANETs enabling optimal route decision or forwarding path selection is of vital significance. Primarily, under mobile network condition where there is significantly high topological variation estimating the BFN is highly tedious task. However, to perform BFN selection under mobility maintaining efficient node table, network and node parameter state information is must. In addition, applying key node information to characterize suitability of a node to become BFN can also play vital role in QoS centric communication. To achieve it, in this research work we assume that each sensor node possesses distinct routing protocol. Being highly dynamic network proactive node management strategy could be applied in which the node parameters could be updated dynamically. It can help in optimal BFN selection process. Since, each node in MANET possesses routing protocol and hence their associated node information from different layers of the protocol stack can be obtained. Considering QoS demands and energy efficiency identifying most significant network parameters such as inter-node link quality, buffer availability at a node, congestion probability at a node, residual energy of a node etc can be applied to assess suitability of a node to become BFN. The selection of BFN based on multiple parameters such as its buffer capacity, packet velocity, link quality, distance etc can ensure optimal forwarding link formation that eventually will help enabling reliable data transmission with minimum data drop probability, minimum deadline miss ratio, minimum latency etc. Furthermore, the use of enhanced SD can enable data type (i.e., RTD, NRT) classification and QoS centric resource allocation. Applying SD to identify type of data under process can help MAC layer and Network layer to perform resource scheduling. Similarly, identifying packet velocity at a node can assist PHY layer to control power management and transmission at physical layer. Similarly, estimating congestion probability and link quality of a node can help MAC layer to avoid that node to ensure reliable data transmission. However, in practice, these parameters are obtained at different layers of the protocol stack and hence sharing this information across the layers can be vital to perform BFN decision. With this motivation, in this research work we have derived a novel and robust cross layer protocol design named QoS oriented CrossSynch Routing Protocol to be used for event driven, mission-critical communication over MANETs (QCSRPM) that incorporates an enhanced Service Differentiation and Resource Allocation (SDRA), Congestion Detection and Proactive Neighborhood Table Management at the Network layer, packet velocity estimation and dynamic link quality estimation at the MAC layer and PHY switching or dynamical transmission control at the PHY layer of the protocol stack to perform optimal routing decision and/or BFN selection. A snippet of the proposed routing model is given in Fig.1.

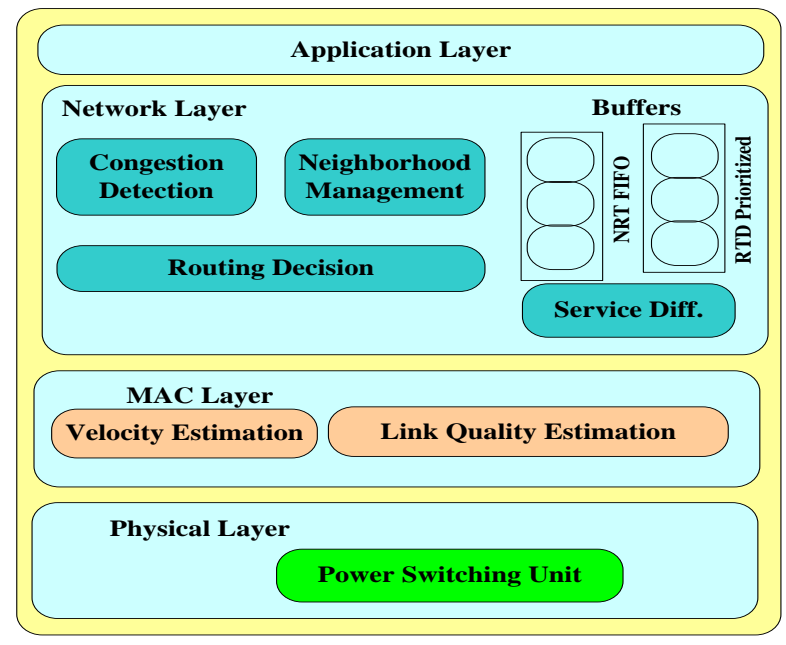

Fig.1. Cross-Synch Architecture based Routing Protocol for MANETs

\section{OUR CONTRIBUTION}

This section primarily discusses the proposed routing protocol and its implementation.

To meet QoS demands, particularly for event driven communication over MANETs identifying Real Time Data (RTD) and Non-Real-Time data (NRT) data is vital 
and furthermore scheduling transmission based on data types is of paramount significance. Identifying the data types and applying appropriate resources or transmission priority can ensure reliable and timely data delivery to make optimal control decisions. Retrieving the key network status (i.e., parameters) from the different layers of the IEEE 802.11 protocol stack can be vital to perform efficient BFN selection that eventually could ensure reliable, QoS centric and energy efficient data communication. In addition, obtaining dynamic network parameters from the different layers of the IEEE 802.11 protocol can be shared with other layers of the protocol stack that could help in performing better routing decision. Such type of protocols are called as Cross-Layer Architecture based protocol that primarily functions by sharing network (state) parameters amongst all layers so as to make synchronized routing decision. CLA concepts have been applied in different routing problems; however very less effort has been made for CLA optimization to be used for MANETs. With this motivation, in this research an enhanced CLA routing model has been developed by considering Network Layer, MAC Layer, and Physical Layer. Unlike traditional approaches where a few parameters such as link quality from the Network Layer or residual energy from PHY layer are used to perform optimal routing decision or BFN selection, Q-CSRPM considers multiple network (node) parameters such as dynamic buffer availability, dynamic link quality (DLQ), packet velocity at a node, and congestion probability at a node etc to perform BFN selection. Q-CSRPM incorporates an enhanced SDFRS model at the Application Layer, Proactive Neighborhood Table Management, Dynamic Congestion Detection (DCD) and enhanced Service Differentiation based Resource Scheduling (SDRS) at the Network Layer, Link Quality Estimation (LQE) and Packet Velocity estimation at the MAC layer, and PHY switching or adaptive rate transmission scheduling at Physical layer of the routing protocol. The use of deadline sensitive data prioritization and transmission scheduling enable proposed method to accomplish reliable and QoS centric event driven communication. Considering real-time application environment, the proposed model is simulated with both RTD and NRT. In conjunction with the dominant QoS demands such as higher throughput, minimum data drop probability, minimum or negligible deadline miss ratio, and optimal bandwidth utilization, Q-CSRPM has exhibited better performance than the other state-of-art routing protocol for MANETs.

The detailed discussion of the proposed Q-CSRPM routing protocol is given as follows:

\section{A. Cross-Synch Routing Model for MANETs}

Considering QoS oriented communication over MANETs, primarily event-driven data transmission, it is inevitable to ensure high Packet Delivery Ratio (PDR), higher throughput, minimum packet drop, low latency and end-to-end delay, and maximum possible resource (i.e., bandwidth) utilization. As already stated in previous section, this research work intends to exploit the dynamic network parameters of MANET obtained from the different layers of the protocol stack (IEEE 802.11) and synchronizing them across the layers to perform reliable and efficient routing decision. As depicted in Fig. 1, different network parameters such as payload types (RTD or NRT), buffer availability (resource available at each node), packet's deadline time, packet velocity, link quality etc are obtained at the different layers of the protocol. Considering parameter's respective significance for a reliable routing model, we have estimated then distinctly at different layers of the IEEE 802.11 protocol stack, which have been further applied to derive a Cumulative Node Rank Matrix (CNRM). Some of the key functional components of our proposed routing algorithm are given as follows:

\section{1) Proactive Node Table Management, \\ 2) Congestion Detection, \\ 3) Service Differentiation and Resource Allocation, \\ 4) Link Quality Measurement, \\ 5) Packet Velocity Measurement, \\ 6) Dynamic Power Management and Physical Switching. \\ 7) QOS oriented Best Forwarding Node Selection in MANETs}

A snippet of the proposed Q-CSRPM routing protocol is given as follows:

\section{1) Pro-active Node Table Management}

As already stated MANETs often undergo topological variations due to node mobility. Under such dynamic network conditions undeniably the node as well as overall network characteristics varies. For example, since each node in the network possesses a fixed buffer space, the buffer availability at a time $t$ may be different than the available resource at $(t+\Delta t)$. It might be because of non-uniform payload arrival from different nodes across network. In other words, during mobility a node might be forced to carry different payloads and therefore it can have different buffer availability at different time. Similarly, due to mobility, the inter-node distance might vary over time. In the same way other key network parameters such as congestion probability, link quality etc. might vary over time in MANETs. Under such situation the static or pre-observed parameters or values can't be optimal to be considered for routing decision over simulation period or application period. Though, network parameters vary over time, updating these parameters pro-actively are inevitable. With this motivation, we have performed proactive node table management that enables dynamic network or/and node parameter update so as to assist reliable BFN selection and routing decision process. In our proposed routing model node parameters are updated dynamically that enables swift and efficient routing decision. Here, each node maintains a node table with multiple blocks to store and update node characteristics such as maximum buffer capacity, current buffer availability, NodeID, node position, current packet velocity, link quality etc. Each 
node updates its node table distinctly by transmitting a Hello or beacon message to one-hop distant neighbor node. The transmission message contains vital node information, including NodeID, current buffer availability, link quality, packet velocity, node location etc. One of the key novelties in our node management scheme is it's avoidance of AKNOWLEDGEMENT message that eventually reduces signaling overheads, energy consumption and latency to a great extent. The applied packetization model is depicted in Fig. 2 .

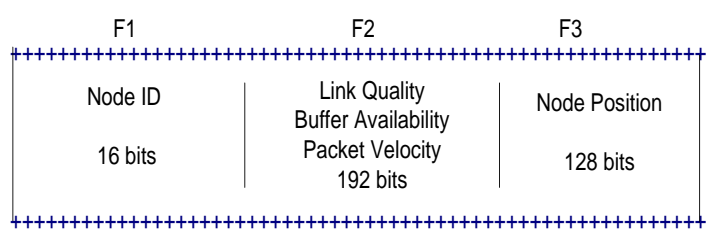

Fig.2. Packet Structure

As depicted in Fig. 2, Q-CSRPM routing model has transmission packet each of 42 byte size. The overall packet is split into three distinct fields; one (16 bits) for NodeID, second field (192 bits) contains node current status such as link quality, current buffer availability, packet velocity etc. The third field (128 bits) contains node location or position information. In practice, once transmitting beacon message to the neighboring node, each node updates its node table. In MANET routing approach one of the key issues is packet collision during transmission. To alleviate such issue our proposed routing model, Q-CSRPM each node multicasts beacon message that operates in conjunction with a random Offset Timer, which is decided based on a normal homogeneous distribution scheme. Retrieving any packet transmission request the node resets its offset timer. The nodes present at one-hop distance with equal or shorter distance to the nearest destination is updated in the table. Let $\mathrm{Neigh}_{\mathrm{j}}$ be the one-hop distant neighbor (node) and $\mathrm{BFN}_{\mathrm{i}}$ be the feasible BFN. As depicted in (1), parameters Eucl $_{\mathrm{F}}$ and Eucl ${ }_{\mathrm{d}}$ signify the Euclidean distance between the BFN and the source node to the nearest sink. In this manner, the node table is updated with nodes $\mathrm{N}_{\mathrm{T}}$ with following conditions (1).

$$
\text { Node }_{\text {Table }}=\left\{B F N_{i} \in \text { Neigh }_{j} \mid \text { Eucl }_{d}-\text { Eucl }_{F} \geq 0\right\}
$$

\section{2) Congestion Detection}

Undeniably, mobility or the dynamic topology in the network often increases the probability of congestion and packet collision during transmission that consequently imposes data drop and retransmission. To alleviate this issue, we have incorporated a timer component. Here, each node multicasts a beacon message controlled through a timer called offset timer. Here, once receiving any transmission request, Q-CSRPM model resets the timer and thus avoids the need of ACK message from the node. Additionally, being a MANET routing approach QCSRPM avoids preserving any path information such as source to destination route information. This is because of node mobility and associated topological variations over simulation period. Under such conditions, a node might receive more payload request to carry relative to its maximum carry capacity or the maximum buffer space. During simulation, the transmission request might vary and accordingly payload in buffer might vary. These events often imposes congestion situation in the network that results into data drop and retransmission. To alleviate such issues, in our proposed model we have applied a dynamic buffer capacity (remaining buffer availability) based congestion detection process at the network layer. Q-CSRPM model exploits the maximum buffer capacity of a node and the current buffer occupied (in other way, remaining buffer) to assess congestion probability at a node in MANET. As stated in above section and Fig. 2, once sending the beacon message a node can obtain the buffer availability of a one-hop neighboring node. Identifying a node with the lower buffer availability than the required buffer, Q-CSRPM routing protocol avoids that node to form the BFN. This as a result reduces probability of data drop and congestion in the network.

To avoid Q-CSRPM routing protocol applies two distinct buffers for RTD and NRT data per node. In our proposed routing protocol, we emphasize on enabling event driven data communication over MANET and therefore RTD data is stored in a prioritized approach while NRT data is stored in a conventional First in First out (FIFO) manner. In event driven communication over MANETs, each packet possesses certain fixed deadline time which is inevitable to make timely control decision. Under such circumstances to enable QoS delivery for event driven communication over MANET, Q-CSRPM protocol provides higher priority to the RTD data, while intending to ensure maximum possible resource provision to the NRT data types. Noticeably, in application RTD data can be certain event data while NRT data might be multimedia data or certain noncritical data. To ensure delay resilient communication we schedule RTD data transmission based on respective deadline time. To achieve it, our proposed routing model considers distance between the source sensor node and the sink. To provide highest priority for RTD data packets it is required to have minimum $\frac{T_{d_{-} i}}{d_{i}^{j}}$, where $T_{d_{-} i}$ states remaining deadline time of the packet and $d_{i}^{j}$ represents Euclidian distance between the forwarding node $\mathrm{i}$ and the nearest sink $\mathrm{j}$. In our work, $T_{\text {Deadline_ } i}$ is estimated by measuring the arrival time of each packet. Here, the value of $T_{d_{-} i}$ is stored for each packets before transmitting packet and associated queuing time is deducted from $\mathrm{T}_{\mathrm{d}_{-} \mathrm{i}}$. To measure the congestion probability at a node, (current) buffer availability information is used. Being a mobile network, MANET's node might undergo dynamic buffer changes that could even give rise to congestion at a node. In our model, a novel parameter named Congestion Index (CI) has been obtained. Here, CI contains node information as well as its relationship with neighboring node in the node subset $\mathrm{S}_{\mathrm{n}}$. Q-CSRPM model estimates the CI value using (2). In Eq. (2), the 
Congestion Index values $\mathrm{CI}_{\mathrm{NRTBuffer}}$ and $C I_{R T D B \text { uffer }}$ signifies the buffer or memory available in NRT related normal queue and the RTD related buffer in prioritized queue, respectively. The other parameters $C I_{\text {NRTBuffermax }}$ and $C I_{\text {RTDMax }}$ signify the maximum buffer capacity of the NRT and RTD data buffers. The total $\mathrm{CI}$ for all the nodes in node set $\mathrm{S}_{\mathrm{n}}$ is obtained as:

$$
C I_{r}=\frac{\mathrm{CI}_{\text {NRTBuffer }}+C I_{R T D B u f f e r}}{C I_{\text {NRTBufferMax }}+C I_{\text {RTDMax }}}+\sum_{i=1}^{N} C I_{r i}
$$

Once estimating buffer availability and congestion of each node, the routing decision can be made to characterize a node to become BFN. Selecting a node with minimum congestion probability would lead reliable and timely data transmission over MANET. In addition, it would reduce congestion probability significantly. In such manner, the proposed model avoids the probability of packet collision too that could reduce retransmission probability and hence energy consumption.

\section{3) Service Differentiation (SD)and Resource Allocation (SDRA)}

In majority of advance communication systems data awareness and associated resource scheduling often play vital role in enabling QoS delivery. Similarly, in MANETs to assure QoS provision, particularly for event driven data communication (i.e., RTD), efficient resource allocation or scheduling is must. Though MANETs could have different types of data to be transmitted and therefore we have considered both RTD as well as NRT data types. Identifying the type of data we have performed optimal resource allocation to each node in the network. In our proposed model, the implemented SD model at first classifies the data into two types, RTD and NRT. We assume that there is the hardware specific provision to identify and classify data into two aforesaid broad types. In our model, RTD data is provided higher priority over normal NRT data to ensure QoS enabled event driven communication over MANETs. In our proposed Q-CSRPM routing protocol each node is assigned its distinct buffer where each node has two distinct buffers for both RTD data as well as NRT data. Noticeably, each buffer (i.e., RTD buffer and NRT buffer) has equal memory or buffer capacity. In this model, in case a node has completely occupied RTD buffer and requires additional buffer to transmit event driven RTD data, then it can borrow the buffer space from NRT buffer where the data are stored in normal FIFO manner. In case, both RTD as well as NRT buffers are complete (i.e., 100\%) occupied, then the proposed SD model drops some of the data elements from NRT buffer. Here, it should be noted that in NRT buffer data packets are stored in normal FIFO manner and therefore dropping a few recently added or queued data packet could not affect overall network performance significantly. Unlike existing SD models where either complete NRT data is dropped to provide additional buffer or external buffer is required to accommodate RTD data, our proposed SD mechanism performs fair resource scheduling while ensuring optimal RTD delivery within deadline time and optimal resource provision to the NRT data. Thus, this approach strengthens our proposed routing model to enables higher resource utilization as well as minimum delay for QoS assurance.

\section{4) Link Quality Measurement}

To ensure QoS centric communication over MANETs, it is inevitable to assess link quality of the nodes so as to characterize its suitability for becoming BFN. Under mobility, the link quality between two nodes might be varying and hence assessing it dynamically can help in reliable forwarding path formation. In this paper, we have applied an efficient dynamic link quality estimation model at the MAC layer of the IEEE 802.11 protocol stack. Q-CSRPM model applies Window Mean Exponentially Weighted Moving Average Algorithm [34] to perform dynamic link quality estimation, where it exploits current packet received ratio parameter. Eq. (3) presents the proposed dynamic link quality measurement $(\psi)$ model.

$$
\psi=\alpha * \psi+(1-\alpha) *\left(\frac{N_{r x}}{N_{t x}}\right)
$$

As depicted in above equation, the packet received ratio which signifies efficacy of the communication link can be applied to characterize the link suitability or BFN. In above equation, the value of $\alpha$ exist in $\alpha \in[0 \ldots 1]$ range. The other parameters $\mathrm{N}_{\mathrm{rx}}$ and $\mathrm{N}_{\mathrm{rx}}$ signify the total number of received packets and the transmitted packets, respectively. Thus, deriving the proposed link quality value (3) each node has been characterized for its suitability to become best forwarding node in MANET.

\section{5) Packet Velocity Measurement}

This is the matter of fact that to ensure QoS delivery over MANETs, it is vital to ensure timely or delay resilient data transmission. In fact, delivering timely data greatly depends on the rate of transmission by a forwarding node. Thus, estimating a node for its current packet velocity can help network controller to perform deadline sensitive data transmission. A node with higher packet velocity can transmit more data in less time that could assist QoS provision and mission critical communication. With this motivation, in our proposed QCSRPM routing protocol, we have estimated packet velocity at each node of the MANET. Q-CSRPM estimates packet velocity by applying key network parameters such as packet delay. Here, packet delay is employed to measure the inter-node distance between neighbor node and the nearest sink. In our proposed routing approach the estimation of (per node) packet velocity enables avoiding the selection of inappropriate node to forward event driven or mission critical communication over MANET. It reduces transmission delay and reduces the likelihood of data drop. In our model, we have applied distance parameter, Euclidian distance in between source and the nearest sink node, relative distance between the neighboring node and the nearest sink, Mean Round Trip Time (MRTT) and the 
speed of radio signal in air. Mathematically, we apply Eq. (4) to derive packet velocity Eq. (5).

$$
S_{t}=\left(\frac{D_{E S D}^{i}-D_{E N S}^{i}}{A R T T_{T i}}\right)
$$

In Eq. (5) the packet velocity $\left(\mathrm{P}_{\mathrm{Vel}}\right)$ signifies the maximum rate of data transmission of a node at a fixed Transmission Power (Tp).

$$
P_{\text {Vel }}=\left(\frac{s_{t}}{R_{\text {Maxspeed }}}\right)
$$

In above equation (Eq. (5)) $D_{E S D}^{i}$ signifies the Euclidean distance between source node i and the sink node, while $\mathrm{D}_{\mathrm{ENS}}^{\mathrm{i}}$ states the distance in between source and the nearest sink. The other parameter $A R T T_{T i}$ and $R_{\text {Maxspeed }}$ states the MRTT value and the highest speed of the radio signal in air. In our model, we used lightspeed as the speed of radio signal. Here, the MRRT value, (i.e., $A R T T_{T i}$ ) is estimated as the difference in time when the packet is transmitted and the ACK is received. We have estimated $A R T T_{T i}$ using Eq. (6).

$$
\operatorname{ARTT}_{T i}=\frac{\sum_{i=0}^{N} R_{A t}^{i}-S_{P t}^{i}}{N}
$$

The parameters $\mathrm{R}_{\mathrm{At}}^{\mathrm{i}}$ and $\mathrm{S}_{\mathrm{pt}}^{\mathrm{i}}$ state the time of ACK receiving and the packet transmission, respectively. Here, the variable $\mathrm{N}$ signifies the total number of packets transmitted.

\section{6) Physical Switching}

In major communication systems augmenting data rate transmission and adaptive control plays decisive role. Controlling network aware packet transmission can not only enable swift data transmission but can also enhance resource allocation. Being cross layer architecture, the proposed routing protocol can apply the network layer and MAC layer information such as packet velocity, buffer availability at a node etc to control dynamic transmission. With this motivation, in this research we have proposed a novel PHY switching model, which might exploit the key network information from upper layer of the protocol stack (IEEE 802.11) to control transmission power and hence rate of the data transmission. This as a result could assure QoS provision for event driven mission critical communication. In addition, more sophisticated approaches such as Dynamic Power Control (DPM) or Adaptive Modulation and Coding (AMC) can also be applied to perform power and/or data transmission control in MANET. Thus, applying above discussed approaches we have performed BFN selection for event-driven communication in MANETs. A brief of the proposed BFN selection model is given as follows:

\section{7) QoS oriented Best Forwarding Node Selection in MANETS}

In our proposed Q-CSRPM routing model multiple network parameters from different layers of the IEEE 802.11 protocol stack have been applied cumulatively to perform QoS centric BFN selection. Here, the predominant motive to use these multiple parameters (i.e. packet velocity, congestion degree of each node, buffer availability, and dynamic link quality) is to ensure reliable forwarding path selection and data transmission. Considering different dynamic network parameters, we have estimated CNRM to assess suitability of a node to become BFN over MANET. Mathematically, CNRM is obtained using Eq. (7).

$$
\mathrm{CNRM}=\mathrm{W}_{1} * \psi+\mathrm{W}_{2} \mathrm{CDC}_{\mathrm{r}}+\mathrm{W}_{3} * \mathrm{PIR}
$$

where, $\mathrm{W}$ states a weight parameter that can be assigned as per specific application need or targeted (focused) parametric value. The other parameter CNRM signifies the cumulative node (i) score matrix, and $\psi$ states link quality. In Q-CSRPM routing protocol the value of $\mathrm{W}$ is assigned in such manner that $\sum_{i=1}^{3} W_{i}=1$. Once estimating CNRM factor, each node is characterized for its suitability to become BFN in MANETs and accordingly the best forwarding path is formed to transmit event driven data over MANETs. The Pseudo code for our proposed Q-CSRPM routing protocol is given as follows (Fig.3).

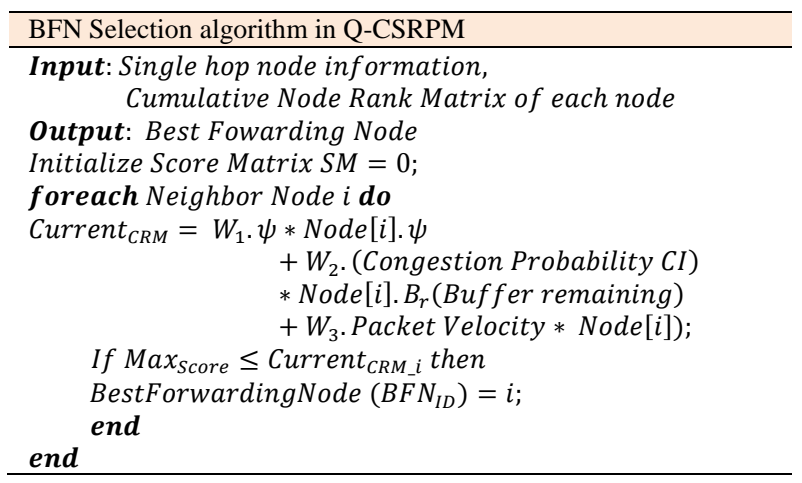

Fig.3. Algorithm for the best Forwarding node Selection in Q-CSRPM

\section{RESULTS AND DISCUSSIONS}

Considering the significance of QoS centric event driven, mission critical communication over MANETs, in this research paper a cross layer model based routing protocol, was developed. Considering node mobility and resulting topological variation in MANETs, our proposed Q-CSRPM considered different network parameters which were obtained from the different layers of the IEEE 802.11 protocol stack to derive a robust routing protocol. Unlike classical approaches, we applied Network layer, MAC layer and Physical layer of the protocol stack and associated network parameter's information was shared amongst different layers to derive a robust best forwarding node selection and path formation. The developed Q-CSRPM protocol performed proactive node management, service differentiation based data prioritization and resource scheduling, and dynamic 
buffer assessment based congestion detection at the network layer, dynamic link quality estimation and packet velocity estimation at the MAC layer and PHY switching control at the physical layer of the protocol stack. Based on the different network parameters such as packet velocity, dynamic link quality and buffer availability (i.e., congestion probability), the best forwarding node selection was performed. Applying aforesaid BFN selection a cumulative node rank matrix (CNRM) was obtained using Eq. (7), where we assigned weight parameters $\mathrm{W}_{1}=0.4, \mathrm{~W}_{2}=0.4$ and $\mathrm{W}_{3}=0.2$. Furthermore, to perform deadline sensitive transmission we assigned initial deadline time per packet as 10 seconds. In this research model to assess efficacy of the proposed Q-CSRPM routing protocol for RTD as well as NRT data delivery over MANETs, we assumed a (hardware) component to classify data as RTD and NRT at the application layer where each node possesses two buffers of equal size. Further, obtaining the different network parameters from the different layers of the protocol stack, CNRM characterized a node to become best forwarding node based on which the best forwarding path was formed for event driven mission critical data transmission. We applied deadline time of each packet to prioritize data transmission. The overall simulation environment considered in this research model is given in Table 2. The overall simulation model was developed using MATLAB 2012b, where to introduce node mobility feature mathematical approach was performed to induce node mobility in circular pattern.

Table 2. Simulation Environment

\begin{tabular}{|l|l|}
\hline \multicolumn{2}{|c|}{ Simulation Environment } \\
\hline OS & $\begin{array}{l}\text { Windows 2010, 4GB RAM, Intel i3 } \\
\text { processor. }\end{array}$ \\
\hline Simulator & Matlab 2012b \\
\hline Network Protocol & Q-CSRPM \\
\hline Data Link & CSMA \\
\hline Physical & IEEE 802.11 \\
\hline MAC & IEEE 802.11 \\
\hline Nodes & Mobile Node-50 \\
\hline Range & $100 \mathrm{~m}$ \\
\hline Payload & $\begin{array}{l}250,500,750,1000,1250,1500,1750, \\
2000,2250,2500,2750,3000 .\end{array}$ \\
\hline $\begin{array}{l}\text { Initial deadline } \\
\text { time(s) }\end{array}$ & 10 Sec. \\
\hline Mobility Pattern & Circular Mobility \\
\hline $\begin{array}{l}\text { BFN Weight } \\
\text { parameters }\end{array}$ & $\mathrm{W}_{1}=0.4, \mathrm{~W}_{2}=0.4$ and $\mathrm{W}_{3}=0.2$ \\
\hline Simulation period & 800 Seconds \\
\hline
\end{tabular}

In the proposed Q-CSRPM model the emphasis was made on embedding major dynamic network parameters and node characteristics to derive a robust route decision model so as to form reliable and QoS centric forwarding path while marinating minimum data drop and maximum resource utilization. As node movement, for simulation circular mobility pattern was considered, where one node tries to forward its data to the next best forwarding node towards destination. To assess efficiency of the proposed Q-CSRPM protocol, a relative cross-layer model, here onwards called existing system [6] was developed. Unlike Q-CSRPM, existing protocol applies only congestion factor to perform route decision over AODV routing protocol. However, authors could not address other key network parameters such as link quality, packet velocity etc to perform routing decision. Even in case of dynamic network conditions, assessing performance while taking multiple network (dynamic) parameters can enable a robust solution. To develop complete simulation environment, MATLAB 2012b software tool was applied. Table II depicts the simulation condition or environment.

For QoS centric and reliable communication for mission critical applications, enabling an algorithm to exhibit RTD delivery before deadline time is inevitable. To achieve it, assuring high throughput or PDR, low packet loss, low deadline miss ratio (DMR) etc is must. Since, retransmission is directly related to the packet drop and therefore reducing packet drop can minimize the probability of retransmission that eventually would assist in reducing energy consumption. Furthermore, assigning sufficient resource for RTD data while ensuring optimal BFN selection based routing can assist delay resilient data transmission. These all factors can significantly enable optimal QoS centric communication over MANETs. With this motivation Q-CSRPM was examined in terms of PDR, packet drop ratio, Deadline Miss Ratio (DMR) for both real as well as non-real time data.

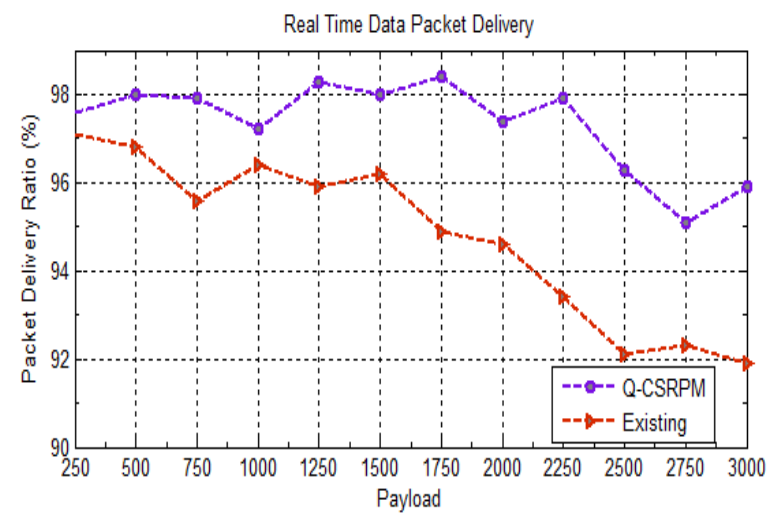

Fig.4. RTD Packet Delivery

To perform comparison of the proposed model a reference work [6] was taken into consideration. Since majority of the MANET routing approaches have applied different variants of AODV protocol for route decision, we considered an enhanced AODV model named Cross Layer based AODV (CLAODV) [6] to assess efficacy of the proposed model. In [6] authors have applied congestion probability of node to perform route decision; however authors could not address other key issues such as link dynamism or dynamic link quality, packet transmission rate or packet velocity etc. to ensure delay resilient transmission over MANETs. Here onwards, we refer the work of [6] as existing system (Fig. 4 to Fig. 8). Fig. 4 presents the PDR performance of the proposed method and the existing system [6]. Here, it can be found 
that over simulation period Q-CSRPM protocol exhibits the highest PDR of $98.2 \%$, which is more than the existing CLAODV [6] (92\%). Here, the role of the proposed multiple parameters based BFN can't be ignored in achieving augmented performance by QCSRPM. Noticeably, Q-CSRPM exhibited better PDR for RTD delivery (98.2\%) as compared to the NRT data (93\%), as depicted in Fig. 5. It can be due to the data drop from FIFO queue in the situation when RTD data required additional buffer to ensure QoS delivery in resource constrained condition. In this situation, some of the NRT packets are dropped that influenced overall PDR. However the results obtained are better than the existing systems [5,6]. In [5] authors have incorporated cross layer model for multipath routing in MANET. Assessing performance, it can be found that our proposed QCSRPM model performs better than the other state-of art techniques $[5,6]$. The average throughput of the existing system was found to be $94 \%$; while the same for the proposed Q-CSRPM was measured as 97\%, which is higher than the CLAODV [6]. It can be because of the enhanced BFN selection and route decision. Other way, the use of multiple network parameters for dynamic best forwarding node selection has enabled proposed method to ensure minimum data drop and hence higher PDR efficiency.

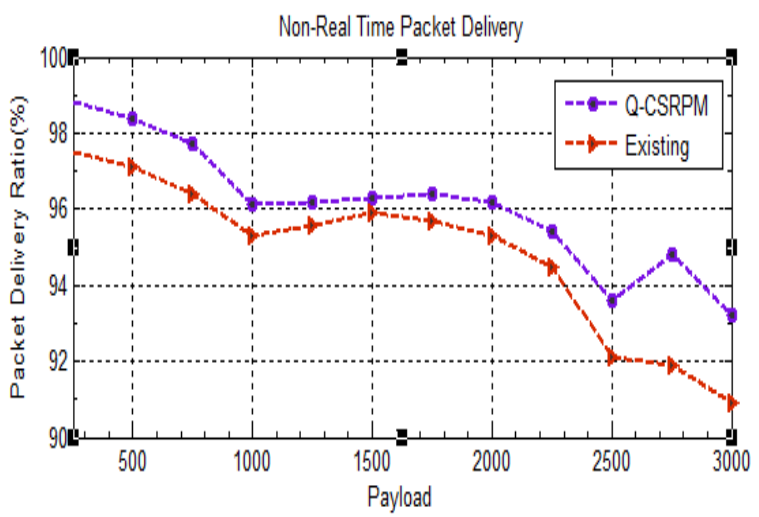

Fig.5. NRT Data Delivery

Fig. 5 presents the PDR for NRT data. Observing the performance (Fig. 5) it can be found that Q-CSRPM routing protocol outperforms existing systems (i.e., CLAODV) even for NRT data types due to enhanced service differentiation based data prioritization and resource scheduling. Fig. 6 and Fig. 7 depict performance in terms of data drop ratio for both RTD and NRT type data, respectively. Results reveal that Q-CSRPM protocol exhibits lower data drop than the CLAODV protocol. Results affirm that the proposed Q-CSRPM shows low packet drop (average 3\%) than the existing system (approximately 6\%). Additionally, it can be found that even increasing the payload, Q-CSRPM performs low data drop. To achieve this result the efficacy of the contribution of optimal buffer provision and adaptive data rate transmission can't be ignored. Considering NRT data transmission performance also, Q-CSRPM has exhibited lower packet drop than the existing system. Even varying the payload, Q-CSRPM routing protocol has exhibited better performance than the existing system. In practice, when payload increases, the probability of congestion too increase and that makes Q-CSRPM to borrow additional space from NRT buffer which reduces the data drop significantly. Here, the use of two distinct buffers; prioritized buffer and the FIFO buffer has helped a lot in bandwidth utilization so as to ensure minimum data drop of NRT while providing best possible resource provision for RTD delivery for event driven communication. The overall results reveal that the proposed Q-CSRPM protocol outperforms existing crosslayer routing approaches.

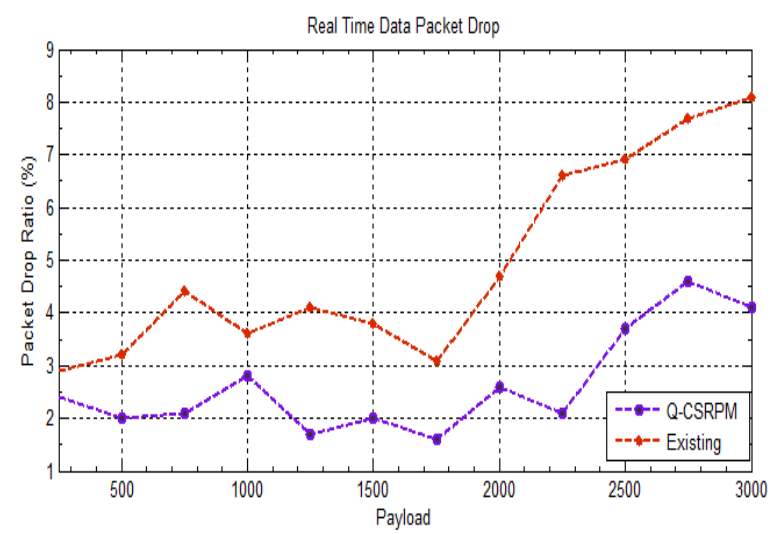

Fig.6. RTD Packet Data Drop

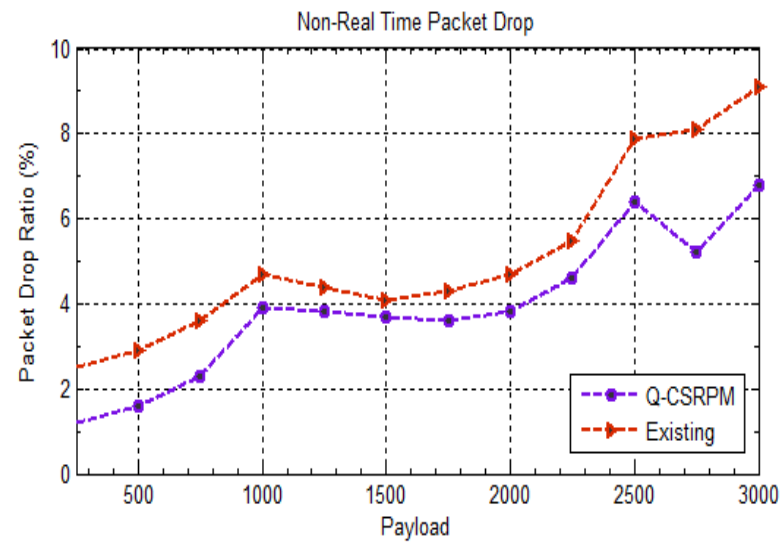

Fig.7. NRT Packet Drop

Fig. 8 exhibits the DMR performance of the proposed Q-CSRPM routing protocol for RTD data transmission over MANET. Q-CSRPM applies deadline time of each data packet as one of the decision variables for BFN selection. In addition, our proposed Q-CSRPM protocol performs deadline sensitive data prioritization and resource allocation that eventually leads reliable data transmission over the proposed routing model. Unlike CLAODV protocol [6] that we had considered as a reference existing system, our proposed Q-CSRPM protocol exhibits better network sensitive routing decision that enables it to exhibit more efficient routing. As already discussed in Section I and III, consideration of a single network parameters such as congestion probability of a node, or inter-node distance or even link quality as individual decision variable can't help enabling reliable routing under dynamic topology. 


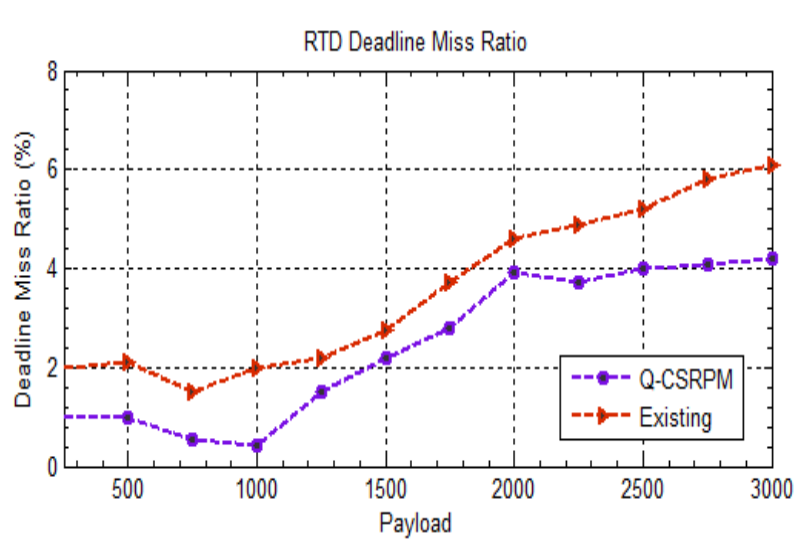

Fig.8. RTD Deadline Miss Ratio with Payload Variation

Referring the existing model, CLAODV protocol [6] that merely applied congestion probability to perform routing decision could have undergone iterative link outage and hence data drop forcing it to undergo data loss and increased delay. This can be easily visualized through the results obtained (Fig. 4 to Fig. 8). On the other hand, the existing system doesn't have any dedicated dual buffer provision for NRT and RTD data, and hence resource scheduling and management is better in our proposed Q-CSRPM protocol. It has enabled achieving higher PDR and low loss rate than the existing CLAODV model. The similar, assessment could be made for the inclusion of packet velocity estimation that enabled our proposed system to assess whether a node could transmit targeted data fast (within remaining deadline time) without imposing deadline miss. It strengthened our proposed routing protocol to assure minimum DMR as compared to the CLAODV routing protocol. The use of deadline sensitive prioritization and transmission scheduling had led minimum DMR that signifies QoS assurance by the proposed Q-CSRPM protocol. The overall results reveal that the proposed cross-layer routing protocol can be of paramount significance for real time, event driven communication over MANETs.

\section{CONCLUSION}

The exponentially rising demand of QoS provision in mobile networks such as MANETs have motivated academia-industries to develop an efficient routing protocol. With this motivation, in this paper a highly robust Q-CSRPM has been developed. The proposed QCSRPM routing protocol applied Application layer, Network layer, MAC layer and the Physical layer parameters to derive a novel routing model. To achieve QoS delivery and energy efficient communication over MANETs, the proposed model implemented Pro-active Node Table Management strategy, dynamic buffer assessment based congestion detection, and enhanced service differentiation and resource scheduling at the network layer, while the MAC layer embodied dynamic link quality measurement and packet velocity estimation. The concept of cross-layer design enabled routing model to share dynamic network conditions or states across the
IEEE 802.11 protocol that eventually lead optimal best forwarding node selection and path QoS centric forwarding path formation. Additionally, this research work proposed service differentiation based non-real time and real time data classification, prioritization and fair resource scheduling which played vital role in enabling bandwidth efficient and reliable event driven communication over MANET. The use of velocity estimation and dynamic link quality estimation strengthened the proposed Q-CSRPM protocol to ensure congestion avoidance, packet transmission control and reliable forwarding path formation, respectively. The use of proactive node management too played vital role in enabling swift route decision and best forwarding path selection. The overall simulation results affirmed that the proposed routing protocol exhibited $98.2 \%$ of the successful packet delivery for real time traffic, while the packet loss was observed merely $1.8 \%$. On the other hand, Q-CSRPM protocol exhibited the highest packet delivery ratio of $93 \%$. It signifies robustness of data prioritization and resource scheduling by proposed service differentiation model. The simulation results also affirmed the minimum deadline miss ratio of $2 \%$ while maximum was obtained at $6 \%$. Though the result signifies satisfactory outcome for real time communication in MANETs, however it can be further enhanced by exploiting residual energy per node as decision variable for best forwarding node selection.

\section{REFERENCES}

[1] S. Corson and J. Macker, "Mobile Ad hoc Networking (MANET): Routing Protocol Performance Issues and Evaluation Considerations", IETF WG Charter, http://www.ietf.org/html.charters/manet-charter.html, January 1999.

[2] M. Conti, Gaia Maselli, G. Turi, S. Giordano "CrossLayering in Mobile Ad Hoc Network Design" IEEE Computer Society, February 2004.

[3] B. C. Villaverde, S. Rea and D. Pesch, "Multi- objective Cross-Layer Algorithm for Routing over Wireless Sensor Networks", Third International Conference on Sensor Technologies and Applications, Athens/Glyfada, Greece, 2009, pp. 568-574.

[4] R.Venkatachalam, Dr.A.Krishnan "Multiple Cross-Layer Design Based Complete Architecture for Mobile Adhoc Networks" (IJCSIS) International Journal of Computer Science and Information Security, Vol. 5, No. 1, 2009

[5] Z. Iqbal, S. Khan, Amjad Mehmood, Jaime Lloret, and Nabil Ali Alrajeh "Adaptive Cross-Layer Multipath Routing Protocol for Mobile Ad Hoc Networks" Hindawi Publishing Corporation Journal of Sensors Volume 2016, Article ID 5486437, 18 pages.

[6] R. Chauhan, P. Saini "Performance Evaluation of Layered and Cross Layered based MANETs" International Journal of Computer Applications (0975 - 8887) Volume 145 No.11, July 2016.

[7] P.A. Abdul Saleem, Dr. Naveen Kumar "Cross Layer Design Approach in Wireless Mobile ADHOC Network Architecture" International Journal of Advanced Research in Computer and Communication Engineering Vol. 2, Issue 3, March 2013.

[8] S. Thacker "Enhancing Routing With Cross Layer Optimization in MANETs" International Journal of 
Computer Science and Information Technologies (IJCSIT), Vol. 5 (3) , 2014, 3708-3710.

[9] D. Chen and P. K. Varshney, "QoS support in wireless sensor networks: a survey", International Conference on Wireless Networks, ICWN '04, Las Vegas, USA, June 2004, pp. 227-233.

[10] F. Xia, "QoS Challenges and opportunities in wireless sensor/actuator networks", Sensors 2008, vol.8, no.2, 2008, pp. 1099-1110.

[11] Y.J. Li, C.S. Chen, Y.Q. Song and Z. Wang, "Real-time QoS support in wireless sensor networks: a survey", 7th IFAC International Conference on Fieldbuses and Networks in Industrial and Embedded Systems, FeT'07, Toulouse, France, 2007.

[12] H. Alwan and A. Agarwal, "A Survey on Fault Tolerant Routing Techniques in Wireless Sensor Networks", in proceedings of the Third International Conference on Sensor Technologies and Applications, Athens/Glyfada, Greece, 2009, pp. 366-371.

[13] K. Sohrabi, J. Gao, V. Ailawadhi, and G. Pottie, "Protocols for self organization of a wireless sensor network," IEEE Personal Communications, vol. 7, no. 5, 2000, pp. 16-27.

[14] K. Akkaya and M. Younis, "An Energy-Aware QoS Routing Protocol for Wireless Sensor Networks", in proceedings of the 23rd International Conf. on Distributed Computing Systems Workshops, 2003, pp. 710-715.

[15] J. Chen, R. Lin, Y. Li and Y. Sun, "LQER: A Link Quality Based Routing for Wireless Sensor Networks", Sensors, vol. 8, 2008, pp.1025- 1038.

[16] Y. Fang, Y. Zhou, X. Jiang and Y. Zhang, "Practical Performance of MANETs Under Limited Buffer and Packet Lifetime," in IEEE Systems Journal, vol. 11, no. 2, pp. 995-1005, June 2017.

[17] Y. H. Chen, E. H. K. Wu and G. H. Chen, "BandwidthSatisfied Multicast by Multiple Trees and Network Coding in Lossy MANETs," in IEEE Systems Journal, vol. 11, no. 2, pp. 1116-1127, June 2017.

[18] Y. Komai, Y. Sasaki, T. Hara and S. Nishio, " \$ $\{\mathrm{k}\} \$$ Nearest Neighbor Search for Location-Dependent Sensor Data in MANETs," in IEEE Access, vol. 3, no. , pp. 942-954, 2015.

[19] R. Jia et al., "Optimal Capacity-Delay Tradeoff in MANETs With Correlation of Node Mobility," in IEEE Transactions on Vehicular Technology, vol. 66, no. 2, pp. 1772-1785, Feb. 2017

[20] A. Taha, R. Alsaqour, M. Uddin, M. Abdelhaq and T. Saba, "Energy Efficient Multipath Routing Protocol for Mobile Ad-Hoc Network Using the Fitness Function", in IEEE Access, vol. 5, no. , pp. 10369-10381, 2017.

[21] S. Sett, P. Kumar Guha Thakurta "Effect of optimal cluster head placement in MANET through multi objective GA", In Computer Engineering and Applications (ICACEA), 2015 International Conference on Advances in, pp. 832-837. IEEE, 2015.

[22] D. Barman Roy Rituparna Chaki "MCBHIDS: Modified layered cluster based algorithm for black hole IDS", In India Conference (INDICON), 2013 Annual IEEE, pp.1-6. IEEE, 2013.

[23] Neha Gupta, Rajeev Kumar Singh, Manish Shrivastava, "Cluster formation through improved weighted clustering algorithm (IWCA) for mobile ad-hoc networks", In Wireless and Optical Communications Networks (WOCN), 2013 Tenth International Conference on, pp. 15. IEEE, 2013.

[24] M. Rath, B. Pati and B. K. Pattanayak, "Cross layer based QoS platform for multimedia transmission in
MANET," 2017 11th International Conference on Intelligent Systems and Control (ISCO), Coimbatore, 2017, pp. 402-407.

[25] M. A. Gawas, L. J. Gudino and K. R. Anupama, "Cross layer multi QoS metric routing for multimedia traffic in 802.11E over MANETs," 2016 Eighth International Conference on Ubiquitous and Future Networks (ICUFN), Vienna, 2016, pp. 582-587.

[26] Z. Li and X. Yang, "A Reliability-Oriented Web Service Discovery Scheme with Cross-Layer Design in MANET," 2016 IEEE International Conference on Web Services (ICWS), San Francisco, CA, 2016, pp. 404-411.

[27] S. V. Sangolli and J. Thyagarajan, "An efficient congestion control scheme using cross-layered approach and comparison of TCP variants for mobile ad-hoc networks (MANETs)," 2014 First International Conference on Networks \& Soft Computing (ICNSC2014), Guntur, 2014, pp. 30-34.

[28] T. Sanguankotchakorn and K. Pudashine, "A cross-layer framework for efficient MPEG-4 video streaming over IEEE 802.11e in MANETs," The 8th Electrical Engineering/ Electronics, Computer, Telecommunications and Information Technology (ECTI) Association of Thailand - Conference 2011, Khon Kaen, 2011, pp. 369372.

[29] V. C. Frias, G. D. Delgado and M. A. Igartua, "Multipath Routing for video-streaming services over IEEE 802.1le Ad hoc Networks," 2006 International Conference on Software in Telecommunications and Computer Networks, Split, 2006, pp. 132-136.

[30] G. D. Delgado, V. C. Frias and M. A. Igartua, "Videostreaming Transmission with QoS over Cross-Layered Ad hoc Networks," 2006 International Conference on Software in Telecommunications and Computer Networks, Split, 2006, pp. 102-106.

[31] X. Wang and J. Li, "Improving the Network Lifetime of MANETs through Cooperative MAC Protocol Design," in IEEE Transactions on Parallel and Distributed Systems, vol. 26, no. 4, pp. 1010-1020, April 2015.

[32] C. T. Calafate, M. P. Malumbres, J. Oliver, J. C. Cano and P. Manzoni, "QoS Support in MANETs: a Modular Architecture Based on the IEEE 802.11e Technology," in IEEE Transactions on Circuits and Systems for Video Technology, vol. 19, no. 5, pp. 678-692, May 2009.

[33] Y. Wei, G. Xie and Z. Li, "A hierarchical cross-layer protocol for group communication in MANET," 2007 IEEE International Conference on Telecommunications and Malaysia International Conference on Communications, Penang, 2007, pp. 62-67.

[34] A. Woo and D. Culler, "Evaluation of efficient link reliability estimators for low-power wireless networks," EECS Department, University of California, Berkeley, Tech. Rep. UCB/CSD-03-1270, 2003.

\section{Authors' Profiles}

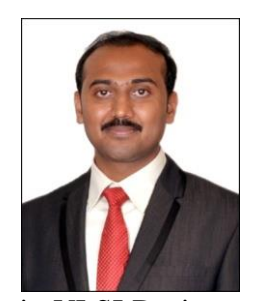

Shashi Raj K. is an Assistant professor in the department of Electronics and Communication Engineering at Dayananda Sagar College of Engineering, Bengaluru. $\mathrm{He}$ has received his B.E degree in Electronics and Communication Engineering from Vivekananda Institute of Technology, Bengaluru and M.Tech degree in VLSI Design and Embedded Systems from Reva Institute of 
Technology and Management, Bengaluru and pursuing Ph.D in Visvesvaraya Technological University, Belgaum, Karnataka, India. He has eleven international, one national journal publications and three international conference publications to his credit. His research area and area of specialization includes Wireless Networks, VLSI and Embedded systems. He is a life member of the Indian Society for Technical Education.

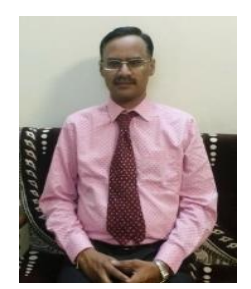

Dr. Siddesh G. K. is a Professor and Head of the department of Electronics and Communication Engineering at JSS Academy of Technical Education Bengaluru. He obtained his Bachelors and Masters Degree in Electronics and Communication Engineering from Bangalore University and Manipal Academy of Higher Education, Karnataka respectively. He also obtained his doctrol degree from Visvesvaraya Technological University Belgaum, Karnataka, India. He has 20 international journal publications and 5 international conference publication to his credit. His research area and area of specialization includes Wireless communication, Computer Networks, Image and Data compression and Processing.

How to cite this paper: Shashi Raj K, Siddesh G K,"QoS Oriented Cross-synch Routing Protocol for Event Driven, Mission-critical Communication Over MANET: Q-CSRPM", International Journal of Computer Network and Information Security(IJCNIS), Vol.10, No.11, pp.18-30, 2018.DOI: 10.5815/ijcnis.2018.11.03 